\title{
Triaging: A New Algorithm for Endoscopy
}

\author{
Ganesh N. Ramesh ${ }^{1}$ \\ ${ }^{1}$ Department of Gastroenterology, Aster Hospital, Kochi, Kerala, \\ India
}

J Digest Endosc 2020;11:44-44

In the setting of a pandemic, there are following three main drivers for provision of health care services: (1) the limited availability of resources (masks, personal protective equipment [PPE], face protectors, etc.), (2) the risk of potential exposure and spread of infection to patients, and (3) the availability of appropriate health care workers (HCWs). A gastroenterologist's role in the pandemic balances between two big challenges: on the one hand to reduce/prevent transmission from asymptomatic or symptomatic novel coronavirus disease 2019 (COVID-19) patients and to protect members of the endoscopy team, and on the other hand, an equally big challenge of meeting the requirements of the non-COVID-19 population with gastrointestinal (GI) problems by virtue of a careful process of selection. This is, indeed, a delicate exercise in decision making and therein lies the importance of "Triaging in the Endoscopy Service." Add to this, the complexities of preprocedure testing and resource allocation, the enormity of this challenge increases several folds.

A very pertinent question that keeps coming up for discussion is "why is the spread of the disease via HCWs such a concern?" The answers, to my mind, are fairly straight forward. If inappropriate utilization of PPE, is one major concern, the shortage of HCWs due to infection and/or quarantine, is an equally big concern. The infected "asymptomatic" HCW as a vector for transmission to patients and to others should probably be the biggest concern, especially in the wake of a community spread.

Gastroenterologist, therefore, is a very crucial link in this chain (as also the pulmonologist), and this is because every
Address for correspondence Ganesh N Ramesh, Department of Gastroenterology, Aster Hospital, Kochi 682027, Kerala, India (e-mail: ganesh.ramesh@rediffmail.com).

endoscopic procedure is potentially aerosol generating, and this includes colonoscopic procedures as well. All patients undergoing endoscopy should be considered potentially infective, unless previously tested.

Lakhtakia and Ramchandani have been very clear and lucid about the science and art of triaging. ${ }^{1}$ Their message is very effectively summed up in the final algorithm, which revolves around four questions as follows:

1. Does the patient have FTOCC (fever, travel history, occupation, contact, and cluster)?

2. Does the patient have respiratory symptoms?

3. The COVID-19 test: not done/positive/negative

4. The type of procedure: emergency/urgent or time sensitive/elective or time insensitive

There are great recommendations to be followed, judiciously and strictly, but some questions still do remain. If the routine procedures are being put off, then for how long? 4 weeks? 8 weeks? 6 months? We don't have the answers yet. What are the parameters, that if fulfilled, will deem it safe for us to come back to normal functioning? Or has COVID-19 the potential to change our practice of endoscopy forever? Only time will tell.

\section{Conflict of Interest}

None declared

\section{References}

1 Lakhtakia S, Ramchandani M. Triaging patient undergoing endoscopy. J DigestEndosc 2020;11;41-43
DOI https://doi.org/

10.1055/s-0040-1712705

ISSN 0976-5042.
License terms

() (1) $\Theta \circledast$ 